\title{
Binaries as Sources of Gravitational Waves
}

\author{
Gijs Nelemans
}

\begin{abstract}
With the discovery of both binary black hole mergers and a binary neutron star merger the field of Gravitational Wave Astrophysics has really begun. The current advanced LIGO and Virgo detectors are laser interferometers that will improve their sensitivity in the coming years. In the long run, new detectors such as LISA and the Einstein Telescope will have sensitivities that allow the detection of many thousands of sources and ET can observe essentially the whole observable Universe, for heavy black holes. All these measurements will provide new answers to open questions in binary evolution, related to mass transfer, out-of-equilibrium stars and the role of metallicity. In addition, the data will give new constraints on uncertainties in the evolution of (massive) stars, such as stellar winds, the role of rotation and the final collapse to a neutron star or black hole. For black hole binaries, the number of detections is rapidly approaching 10 and the first proper statistical studies of the population can be done soon. In the long run, the thousands of detections by ET will enable us to probe their population in great detail over the history of the Universe. For neutron stars, the first question is whether the first detection GW170817 is a typical source or not. In any case, it has spectacularly shown the promise of complementary electromagnetic follow-up. For white dwarfs we have to wait for LISA (around 2034) but new detections by e.g. Gaia and LSST will prepare for the astrophysical exploitation of the LISA measurements.
\end{abstract}

\subsection{Gravitational Waves and their Detection}

With the discovery of first the binary black hole merger GW150914 (Abbott et al., 2016e) and recently the binary neutron star merger GW170817 (Abbott et al.,2017ef), the field of Gravitational Wave (GW) astrophysics has been opened in a spectacular fashion.

Since the original prediction of GW (Einstein, 1916, 1918), the field has made a long journey. Since the 1960s, the importance of GW for binary stars and the importance of binaries as GW sources has been recognised (e.g. Paczyński, 1967; Press and Thorne, 1972). Since the 1970s, binary stars, in particular those with neutron star (NS), white dwarf (WD)

This material has been published in The Impact of Binaries on Stellar Evolution, Beccari G. \& Boffin H.M.J. (Eds.). This version is free to view and download for personal use only. Not for re-distribution, re-sale or use in derivative works. (C) 2018 Cambridge University Press. 
and (hypothetical) black hole $(\mathrm{BH})$ components, have been considered the most promising sources for detectable GW (e.g. Clark et al., 1979; Tutukov and Yungelson, 1979, Nelemans et al., 2001; Belczynski et al., 2002). With the development of laser interferometer detectors such as LIGO and Virgo, the field gradually evolved to the stage were real detections of GW on Earth could be expected. At the same time, a long history of proposed GW detectors in space has culminated in the selection of the LISA mission as the first space detector (Amaro-Seoane et al. 2017).

For excellent reviews about GW and GW sources I refer e.g. to Schutz (1989); Sathyaprakash and Schutz (2009). For binaries, the effect of GW emission is a decrease in orbital energy and angular momentum (e.g.Peters, 1964) leading, in general, to a shortening of the orbital period and circularization of the orbit. This will stop once the stars start to interact which, in most cases, leads to merger of the two stars. Far from the sources, the effect of GW is a minuscule effect on the metric, that can in principle be measured.

\subsubsection{Detectors: Status and Future}

The way the minute ripples in space time can be measured is essentially by determining the time difference that a light ray spends traversing a "fixed" distance using laser interferometry (Weiss, 1972). This clever idea, and the skills and endurance to turn the idea into a working detector was awarded the 2017 Nobel Prize in Physics for Weiss, Thorne and Barish. GW come with different frequencies/wavelengths (depending on the size of the source) and detectors need to be properly sized to be sensitive to certain waves (very roughly source size $\approx$ wavelength $\approx$ detector size).

The currently operational ground based laser interferometers advanced LIGO (Aasi et al. 2015; Abbott et al., 2016c) and advanced Virgo (Acernese et al., 2015) are 4 and $3 \mathrm{~km}$ long vacuum instruments in which laser beams are first accurately stabilised (both in frequency and in power) and then split over the long arms. In the arms the lasers are reflected hundreds of times in Fabry-Perot cavities, increasing the effective size of the detector, before being combined again and tuned in such a way that almost perfect destructive interference is achieved. Since this means most of the light is reflected back towards the laser, a power-recycling mirror is placed in the path of the laser, reflecting the light back into the interferometer, vastly increasing the power of the light stored in the interferometer and thus increasing the sensitivity of the instrument. This is needed because the length changes are so small that they are an incredibly small fraction $\left(\sim 10^{-10}\right)$ of the $1 \mu$ wavelength of the laser and thus lead to only tiny changes in the interferometer power output.

In addition to achieving the necessary accuracy of the measurement, the detectors need to be isolated from environmental disturbances and carefully monitored to determine any external noise components. Seismic noise is the most important noise source at low frequencies and is suppressed by many orders of magnitude via seismic isolation mechanisms. After all these measures, there are still large numbers of transient noise fluctuations that can mimic real GW signals, collectively know as triggers. By searching for coincident and identical triggers in multiple detectors, the trigger background can be very strongly 
reduced. However, even then the number of triggers increases very sharply when going to weaker triggers, so there is a fairly clean separation between noise and real signals (see e.g. fig 4 of Abbott et al. 2016e).

The two advanced LIGO and the advanced Virgo detectors are currently offline to undergo further improvement to increase the sensitivity. The plan is for the detectors to come online again for the year-long third observing run (O3) about a year after the end of $\mathrm{O} 2$ (Abbott et al. 2016f), i.e. in the fall of 2018/beginning of 2019. After that, further improvements are expected before the detectors reach their design sensitivity. Additional detectors, KAGRA in Japan (Aso et al. 2013) and LIGO India (Iyer et al. 2011) are expected to join the GW detector network in the early 2020s. Further improvements on the sites of the current detectors are envisioned in the later 2020s (termed A+ and LIGO Voyager for LIGO sites).

On a longer time scale a third generation of detectors is planned, such as the European Einstein Telescope or the US Cosmic Explorer, each with significantly larger arms (10$40 \mathrm{~km}$ ), possibly underground to reduce the environmental noise. The sensitivities of these detectors would be at least a factor 10 better than the advanced detectors (e.g. Sathyaprakash et al. 2012). Boosted by the success of the second generation detectors, the preparations for the third generation detectors is taking off $a$

For larger GW wavelengths the ESA LISA mission is planned for a launch in 2034 (Amaro-Seoane et al., 2017) . It consists of three identical space craft that exchange laser beams in an interferometer with arms lengths of 2.5 million $\mathrm{km}$. Although the basic measurement idea is the same as for the ground based detectors, it is not possible to actually reflect the laser beam back along the arms, since the divergence of the laser beam would lead to a signal that is much too weak. Instead the technique of Time Delay Interferometry (Armstrong et al. 1999) is used, in which at each time and in each of the spacecraft the phase information of the incoming laser beam is measured by comparing it to a local reference. All this information is later combined into a virtual interferometer by adding up signals with the appropriate time shifts to determine the interference that would have resulted if the light had actually followed that path and would have been physically combined. The advantage of this technique is that the laser noise largely cancels and that different types of virtual interferometers can be constructed (referred to as different TDI configurations/variables). The LISA mission is currently studied by ESA with a significant contribution from NASA and the final adoption of the mission is expected in the early 2020s.

\subsubsection{Order of Magnitude Estimates: Detection Horizons}

Gravitational waves are produced by the sources with a changing quadrupole moment and thus binary stars are about the most optimal sources for GW generation (e.g. Thorne, 1997). There is a nice paradox that GW are energetically at the same time the weakest and the 


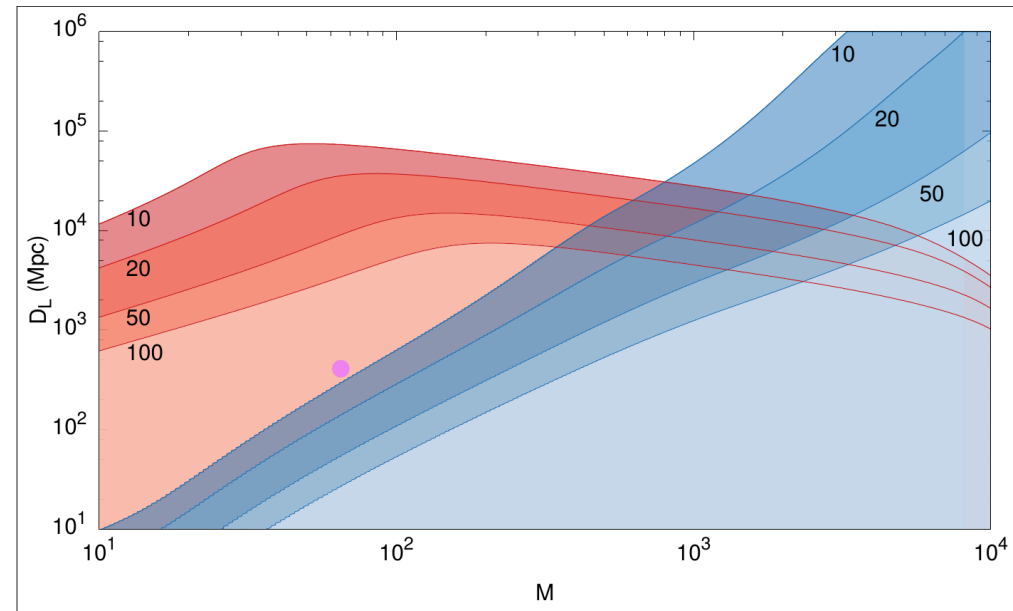

Figure 1.1 Detection horizons as s function of total mass for equal mass binaries for future detectors. The expected S/N is given by the contours. GW150914 is indicated by the dot. Figure courtesy Neil Cornish

strongest phenomena in the Universe. The GW luminosity of a binary GW source is given by (Peters and Matthews, 1963)

$$
L_{\mathrm{GW}}=\frac{32 G^{4}}{5 c^{5}} \frac{M_{1}^{2} M_{2}^{2}\left(M_{1}+M_{2}\right)}{a^{5}}
$$

which due to the extremely small pre factor $G^{4} / c^{5} \sim 10^{-81}$ vanishes almost completely for any human-made experimental set-up and even for normal binary stars is very small (e.g. the GW luminosity of Algol is $\sim 10^{-6}$ times the solar luminosity). On the other hand (forgetting factors of a few difference between $M_{1}, M_{2}, M_{1}+M_{2}$ ), the same equation can be written as

$$
L_{\mathrm{GW}} \approx \frac{32 c^{5}}{5 G}\left(\frac{G M}{c^{2} a}\right)^{5}
$$

which can approach $c^{5} / G \sim 10^{59} \mathrm{erg} / \mathrm{s}$, for systems with $v \approx c$, i.e. $G M / a \approx c^{2}$ can thus can reach above $10^{26}$ solar luminosities! This condition $(v \sim c)$ is of course quite extreme, but for binary BHs that are about to merge can be approached.

The frequency of the emitted GW is simply related to the orbital frequency $f_{\mathrm{GW}}=$ $2 f_{\text {orb }} \sim G M / a^{3}$. For merging black holes, the separation at merger scales with the horizon size and thus mass $(a \sim R \sim M)$, i.e. $f_{\text {merge }} \sim 1 / M^{2}$ : more massive BHs merge at lower frequencies.

The (instantaneous) amplitude $h$ of the GW scales with $\sqrt{\left.L_{\mathrm{GW}}\right) /\left(f_{\mathrm{GW}} d\right)}$ and thus $h \propto$ $\mathscr{M}^{5 / 3} f_{\mathrm{GW}}^{2 / 3} d^{-1}$, with "chirp mass" $\mathscr{M}=\left(M_{1} M_{2}\right)^{2 / 5} /\left(M_{1}+M_{2}\right)^{1 / 5}$ and $d$ the distance. We thus can immediately see that for a binary that is not changing its frequency during the observation, the signal scales with the (chirp) mass to the power $5 / 3$, while for such a source 
the signal becomes stronger for higher frequency. For systems that do evolve, such as the observed LIGO/Virgo sources, this picture is not correct, since there is a limited number of GW cycles observed, which is inversely proportional to $\mathscr{M}^{5 / 3}$. The signal strength increases with the square root of the number of cycles, so scales with $\mathscr{M}^{5 / 6}$.

In Fig. 1.1 the distance to which binaries can be observed with ET and LISA is shown as a function of total mass (assuming equal mass binaries). The plot roughly agrees with the scalings derived above, except for masses above several tens of solar masses in ET, where the signals slowly move out of the ET sensitivity band (and the maximum distance thus starts to go down again with mass). Because GW get redshifted, the maximum sensitivity corresponds to increasingly lower mass systems at larger distances. From this figure, the enormous promise of GW astrophysics is immediately clear, since BH binaries can be observed essentially throughout the whole universe with these instruments!

\subsection{What can we Learn about Stars and Binaries from GW Measurements?}

For many people, GW detections are primarily interesting from the point of view of testing General Relativity (Abbott et al., 2016g), having independent cosmological measurements (Abbott et al. 2017a) or detecting signals from the Early Universe or from unknown sources. For us, GW measurements are a new way to study binary systems with compact objects, for which there are many open questions. After listing the most important open questions, I will discuss what we know and can learn about these questions from GW observations.

\subsubsection{Open Questions about Compact Objects}

Compact objects form at the end of the lives of stars. In this way, stellar evolution and compact object formation are intimately linked. If we want to understand the formation of NSs and BHs, we have to know how the massive stars evolve from which they form (e.g. Sukhbold et al. 2016, and chapter 9-11 of this volume). The most pressing issues are stellar winds and the influence of rotation on the internal evolution of the stars. But even if these were known, it is not clear how the final state of the star translates into the formation of the compact object. The way the core collapses and the possible ensuing supernova (SN) explosion are still very unclear as are the resulting masses and spins of the compact objects (e.g. Ugliano et al., 2012). From NS observations it is clear that the collapse leads to an asymmetric kick(e.g. Lyne and Lorimer, 1994). The open question is if this also holds for BHs(e.g. Repetto et al. 2017). All of this is further complicated by binary interactions that lead to mass transfer and influence the evolution of the stars. In addition, the binary evolution processes influence the further evolution of the system and are typically rather poorly understood (mass transfer and its resulting loss of mass and angular momentum from the system, including common-envelope evolution, e.g. chapters $1,4,7,8,12,13$ 


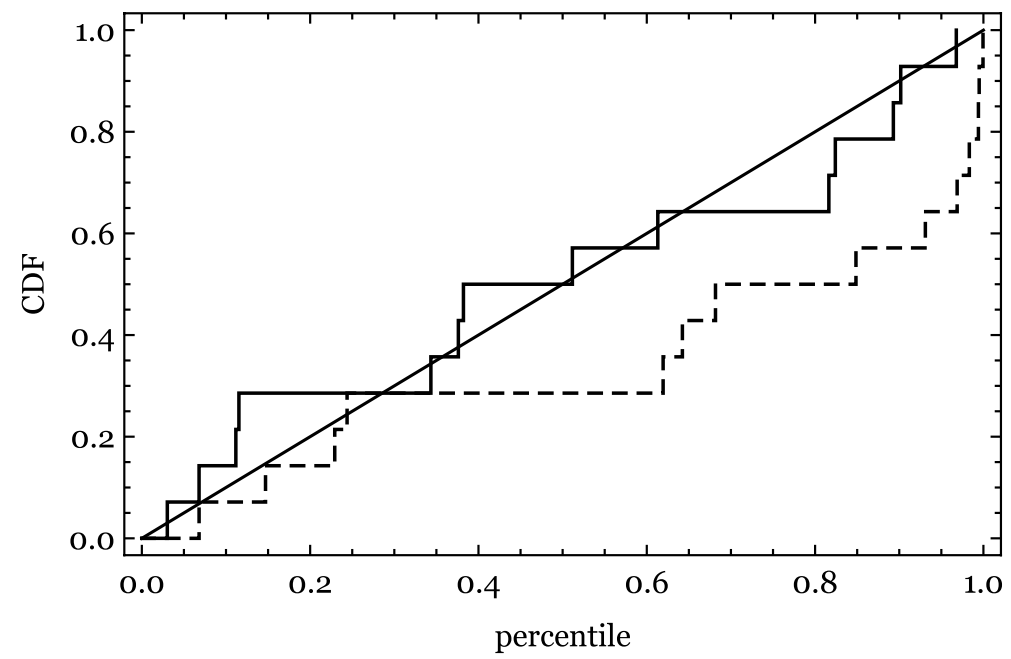

Figure 1.2 Cumulative distribution of the local height above the plane of black-hole X-ray binaries compared to a simulated population that originates in the disk with low (dashed) and high (solid) kicks. From Repetto et al. (2017)

of this volume). Furthermore, if we want to understand compact objects and binaries in the global context of the evolution of galaxies including the Milky Way, we also have to understand all these processes in the changing environment, in particular metallicity. With the new tool of studying compact objects in binaries with GW, we can hope to shed a new light on the combined effect of all the uncertainties and thus learn about them.

\subsubsection{Black Holes}

BHs are the most enigmatic objects, not only by their nature, but also in their formation. We simply do not know which stars form BHs and if this happens via initial formation of a neutron star that later collapses or not and how often (if at all) BH formation is accompanied by a SN (e.g. Ugliano et al., 2012).

A particularly important question is the existence of asymmetric kicks at the formation of BHs. Several individual black-hole X-ray binaries have been studied in detail in order to investigate if proof can be given that BH kicks exist (e.g. Nelemans et al., 1999; Willems et al., 2005; Fragos et al., 2009). Although kicks are possible or even plausible, there is not unambiguous evidence. An alternative approach is to look at the positions of the observed black-hole X-ray binaries in the Milky Way and compare them to those of neutron-star X-ray binaries (White and van Paradijs, 1996, Jonker and Nelemans, 2004; Repetto et al. 2012). The more recent of these studies show that the black-hole systems are found at similar height above the plane as NS systems or at least surprisingly high up if BHs do not receive significant $(\sim 100 \mathrm{~km} / \mathrm{s}$ ) kicks. However, NS systems may have formed in a 


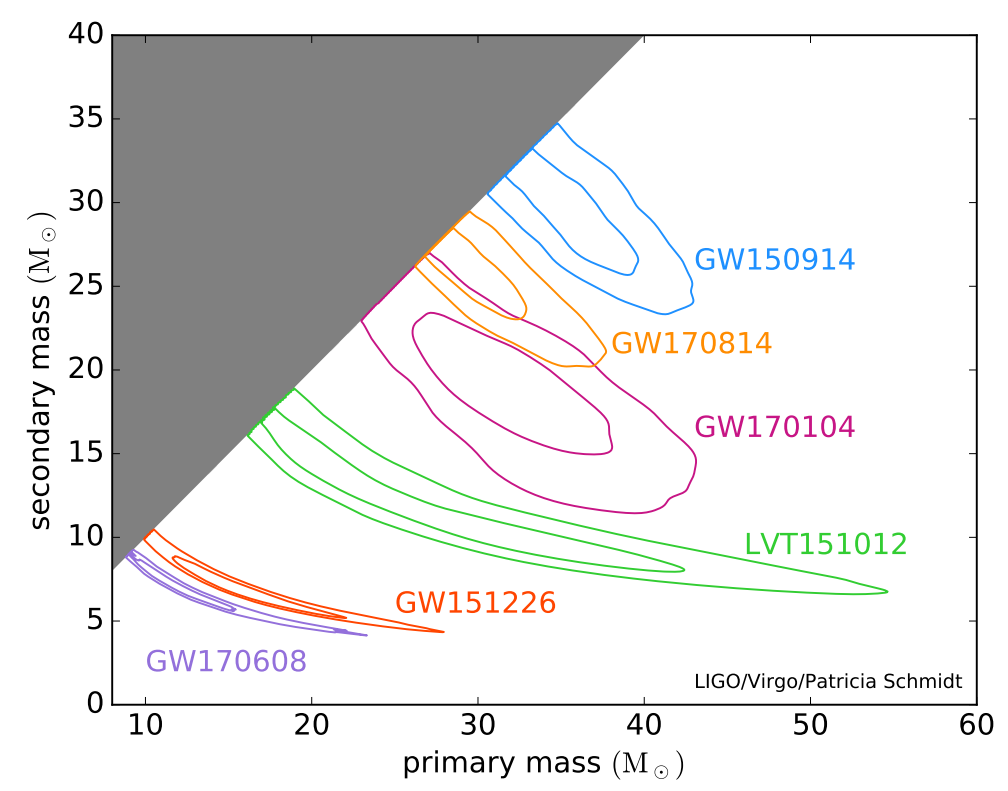

Figure 1.3 Mass measurements of the current binary BH GW detections. Courtesy LIGO/Virgo collaboration/Patricia Schmidt

different way. Also, the observed black-hole systems are differently biased because the companion stars have to be studied in detail in order to show that the compact object is so massive that it has to be a BH. In a systematic study all these effects are taken into account and the local height of BH X-ray binaries is compared to a simulated population for different kicks and rescaled to the local Galactic potential such that all systems can be compared (Repetto and Nelemans, 2015; Repetto et al., 2017). The result is shown in Fig. 1.2 and suggests that indeed at least some BH get relatively high kicks.

At the same time, simulations of the formation of binary BH as GW sources suggest that (too) large kicks disrupt too many systems, leading to a very low merger rate (e.g. Belczynski et al. 2016a). The LIGO/Virgo detections have spectacularly opened a new way to (statistically) study the BH population. Including the most recent announced detection, GW170608, five high-confidence detections and one candidate (LVT151012) detection have been reported (Abbott et al., 2016b, 2017c b d ). They span a wide range of masses (Fig. 1.3 ranging from "heavy" $\mathrm{BH}$ with masses over $30 \mathrm{M}_{\odot}$ to ones in the range of masses known from X-ray binaries (Casares and Jonker. 2014). The rapid increase in sources makes for an exiting expectation that soon we will be able to put real constraints on the mass distribution of binary BH. Looking at Fig. 1.3 it will be the question whether there is a bi-modal distribution with low and high mass systems, or a continuum, especially with the uncertain character of the intermediate system LVT151012.

Apart from the masses, the observations have made it possible to estimate the binary 


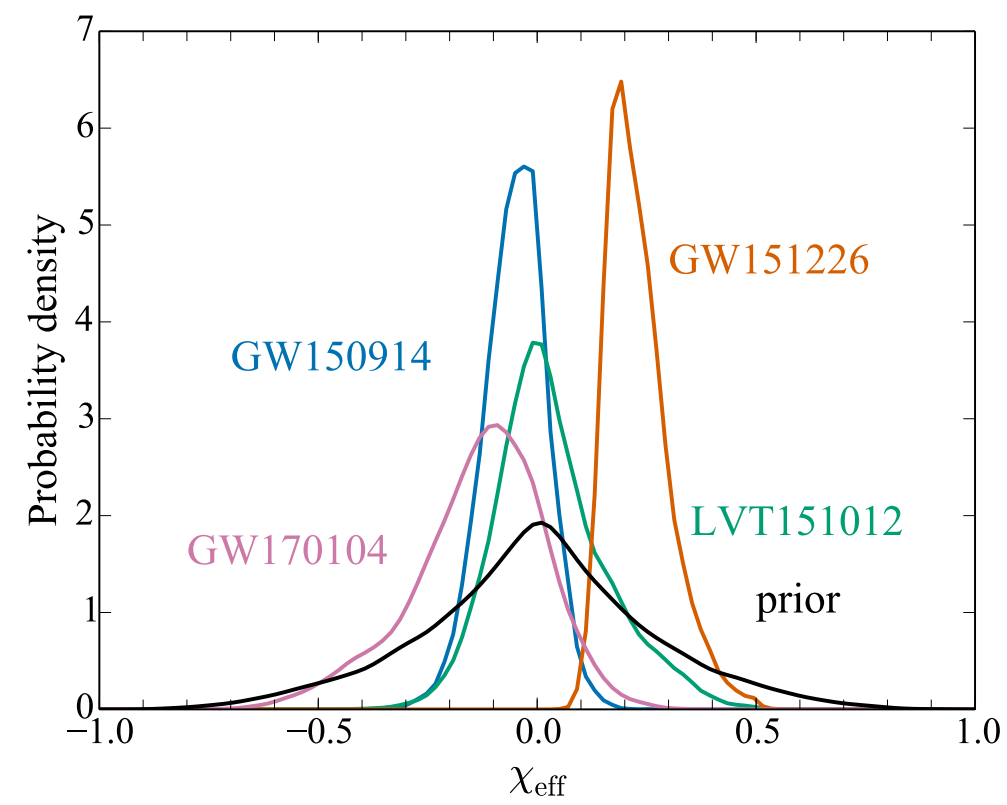

Figure 1.4 Effective spin measurements of the current binary BH GW detections. From Abbott et al. (2017b)

$\mathrm{BH}$ merger rate. All detections, including the newer ones are consistent with the latest rate determination (Abbott et al. 2017b) of $12-213 / \mathrm{yr} / \mathrm{Gpc}^{3}$. The last piece of information that is available from the data is en estimate of the "effective spin", i.e. the mass weighted component of the spin parallel to the orbital angular momentum, see Fig. 1.4 As can be seen, most of the systems have effective spins close to zero.

In our current understanding, there are several ways in which binary $\mathrm{BH}$ that merge can form (apart from "exotic" scenarios such as primordial BH, pop III stars etc) see Abbott et al. (2016a). The first is the same path that has been put forward for the double NS systems found in the 1970s (e.g. Flannery and van den Heuvel, 1975) but then for more massive stars (e.g. Belczynski et al. 2016a b). There are several variations on this scenario, in particular avoiding the common-envelope stage, either by invoking stable mass transfer, possible due to the large masses of the $\mathrm{BH}$ (van den Heuvel et al., 2017) or due to the rapid rotation of the massive stars, leading to so-called homogeneous evolution, in which expansion to giant dimensions is prevented by enhanced mixing (de Mink and Mandel, 2016). A fundamentally different formation scenario is via dynamical interactions in dense stellar environments (Sigurdsson and Hernquist, 1993; Portegies Zwart and McMillan, 2000) that is found to be a viable mechanism to produce mergers such as GW150914 (e.g. Rodriguez et al., 2016, Mapelli, 2016, Park et al., 2017). There is a lot of excitement about the idea that the effective spins will be the key to distinguishing different formation channels or 
inferring BH kicks. The idea is that for dynamically formed systems the angles between the BH spins would be random, while for binaries they would be more or less aligned, unless the BH get very large kicks. However, as long as there is no clear indication that the $\mathrm{BH}$ spin is directly related to the spin of the progenitor star these considerations are not very convincing. The reason to doubt the relation between progenitor spin and $\mathrm{BH}$ spin is the observed very large misalignment between the spins in the double pulsar (Breton et al. 2008) .

Another extremely nice and promising result is the realisation that GW150914-like sources are also promising LISA sources (Sesana, 2016). Apart from a multitude of nice measurements when combining LISA and ground based measurements, there is some chance to detect any remaining eccentricity in the orbits in the LISA band, that would point towards dynamical formation (e.g. Breivik et al. 2016).

It is clear that in the next years we will have a steady increase in the number of detected binary BHs. With improved detector sensitivity, first with the current second generation of detectors and of course more spectacular with the third generation detectors, the numbers could start to add up to very significant numbers. This will allow fantastic studies. My personal expectation is that with several tens of detections we we will get a more solid view of the mass and spin distributions, maybe seeing the first evidence for different populations if they exist. With several hundreds of detections we can start to bin up the measurements, for instance in redshift bins and study the evolution of the system properties. At the same time, the overall mass and spin distributions, including e.g. mass gaps, upper and lower limits to BH masses etc., will be determined quite accurately. Finally, for the third generation detectors, with thousands and thousands of detections, we can really determine all these properties as a function of the evolution of the universe!

\subsubsection{Neutron Stars and Electromagnetic Follow-Up}

Since the discovery of PSR J1913+16 (Hulse and Taylor. 1975), and maybe even before, double NS have been the prime example GW sources (see e.g. Phinney, 1991). The discovery of the orbital change due to GW (Taylor and Weisberg, 1982) started a process that recently reached a spectacular high light with the discovery of the first double NS GW source GW170817, that also has been detected in gamma-rays, optical/infrared, X-rays and radio emission (see Abbott et al., 2017f e).

The current knowledge about the 15 Galactic double NS is nicely summarised in Tauris et al. (2017). From these systems, it is clear that there is a sizable population of double NS that will merge in the Galaxy. The source properties, in particular the fact that at short orbital period there are systems with low and with high eccentricities, suggest that there may be more than one way to form them. It has been suggested, that the low eccentricities arise from NS with small kicks, formed in electron-capture SN (Podsiadlowski et al. 2004) that occur much more frequently in close binary systems than in single stars or wide binaries. However, the recent finding that even a significant fraction of normal radio pulsars has low 


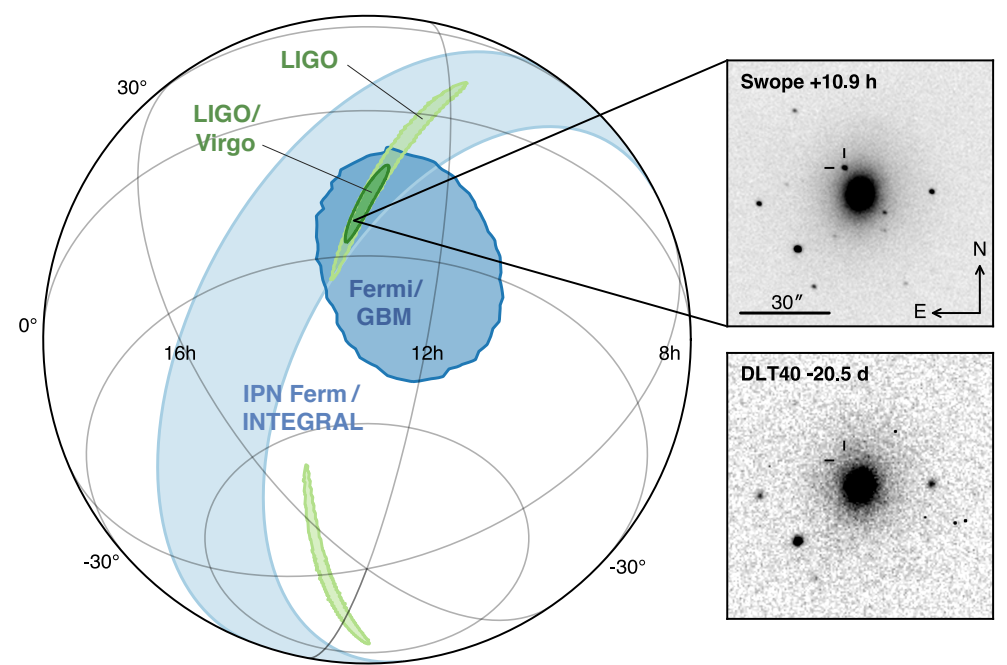

Figure 1.5 Fermi, Integral and LIGO/Virgo localisation of the GW170817 and the subsequent detected optical counterpart. From Abbott et al. (2017f)

velocities (Verbunt et al. 2017) raises some questions about the one to one link between low kicks and electron capture SN.

The formation of double NS has been outlined already in the 1970s (Flannery and van den Heuvel, 1975, De Loore et al., 1975; Massevitch et al., 1976). It starts with two massive stars in which, after a phase of mass transfer, the primary explodes in a SN. If the orbit remain bound, the system temporarily takes the form of a High-Mass X-ray binary in which the NS accretes from the stellar wind of the companion after which it goes through a second phase of mass transfer that leads to strong spiral in (van den Heuvel and De Loore. 1973). The resulting close binary of a helium burning star and a NS may become an X-ray binary again (like Cyg X-3) after which the helium star explodes to become the second NS. $\mathrm{GW}$ emission subsequently brings the system to merger.

The expectation, based on simulations, that after the merger of two NS, some of the material is ejected and the possibility that in the merger (temporarily) a massive, rapidly spinning NS is formed, have led to the suggestion that the merger of two NS should be accompanied by an electro-magentic signal. In particular, the radioactive decay of the ejected low-density NS material, expected to have a brightness in between novae and $\mathrm{SN}$, is often called kilonova or macronova (see Metzger 2017, for a review). The basic physics is very elegantly described in Li and Paczyński (1998) and Kulkarni (2005) and shows that the electro-magnetic signal of the expanding ejecta essentially depends on the injected energy and the opacity of the material. Barnes and Kasen (2013) showed that the opacity of the material may be much higher than Thomson scattering. For still relatively low opacities, the emission comes out early and fast and is blue; for high opacities the emission comes out later and slower and is red. 
In order to fully exploit the promise of multi-messenger GW astronomy, the GW signals need to be quickly identified and shared with EM partners. The experience in the first observing run (Abbott et al. 2016d) has been used to improve the procedures and after the discovery of GW170817 the alert went out within 1 hour. In the first day, several tens of observatories have reacted and observed the counterpart in all bands (see Abbott et al., 2017f, and Fig. 1.5. The optical/infrared counterpart is consistent with the predictions of the kilonova models (Smartt et al., 2017; Cowperthwaite et al., 2017; Pian et al., 2017; Drout et al., 2017; Kasliwal et al., 2017; Levan et al., 2017) although the bright transient implies a quite high ejecta mass $\left(\sim 0.03 \mathrm{M}_{\odot}\right)$. The masses inferred from the $\mathrm{GW}$ detection are similar to the ones of the know double NS (Abbott et al., 2017e).

Even though it is only one detection, it can serve to make an estimate of the merger rate of double NS: $1540_{-1220}^{+3200} / \mathrm{Gpc}^{3} / \mathrm{yr}$. Although within the range of expectations (e.g. Abadie et al. 2010), it is quite high compared to the recent theoretical models and not easy to reconcile with the models without overestimating the binary BH merger rate (Chruslinska et al. 2017). This means that the next observing run will very quickly show if this single detection was a statistical outlier, or if the merger rate really is so high.

With this in mind, and the question whether the high ejecta mass and thus bright kilonova are typical or not, it is important to systematically investigate the GW error boxes in the future. For that, for instance the BlackGEM telescope array is developed. It consists of three $65 \mathrm{~cm}$ telescopes, each with a 2.7 square degree field of view that can reach mag 23 in 5 min integration (Bloemen et al., 2016).

For double NS the expectation is that LISA will observe these systems only in the Milky Way, so expected numbers are low (e.g. Nelemans et al. 2001). However, a very nice feature is that the GW strength of the double NS with periods shorter than about $30 \mathrm{~min}$ is such that the measurement of the population in that period range will be complete (eLISA Consortium et al., 2013).

Overall, the promise of GW astrophysics for the study of NS binaries lies mainly in the determination of the rates and properties of the systems that can be related to the preceding binary evolution. In particular if also EM signals are detected, the study can be extended to included detailed studies of the parent population and the ages of the mergers, probing the delay time between the formation of the double NS and the merger.

\subsubsection{White Dwarfs and Electromagnetic Data}

For WD the LIGO and Virgo detectors are too small. Indeed, any ground based detector will have difficulty detecting any WD, so we have to turn to LISA. It was realised early on (Evans et al. 1987) that double WD would form a large population of detectable sources and even so large that they could form a confusion noise, or foreground noise (e.g. Hils et al. 1990). These systems are now found regularly optically (e.g. Marsh, 2011; Brown et al. 2017). With the current design of the mission (Amaro-Seoane et al. 2017), the sensitivity of LISA is such that with a recent model for the Galactic population of double WD (Toonen et al. 2012) the expectation is that it will detect this foreground, in addition 


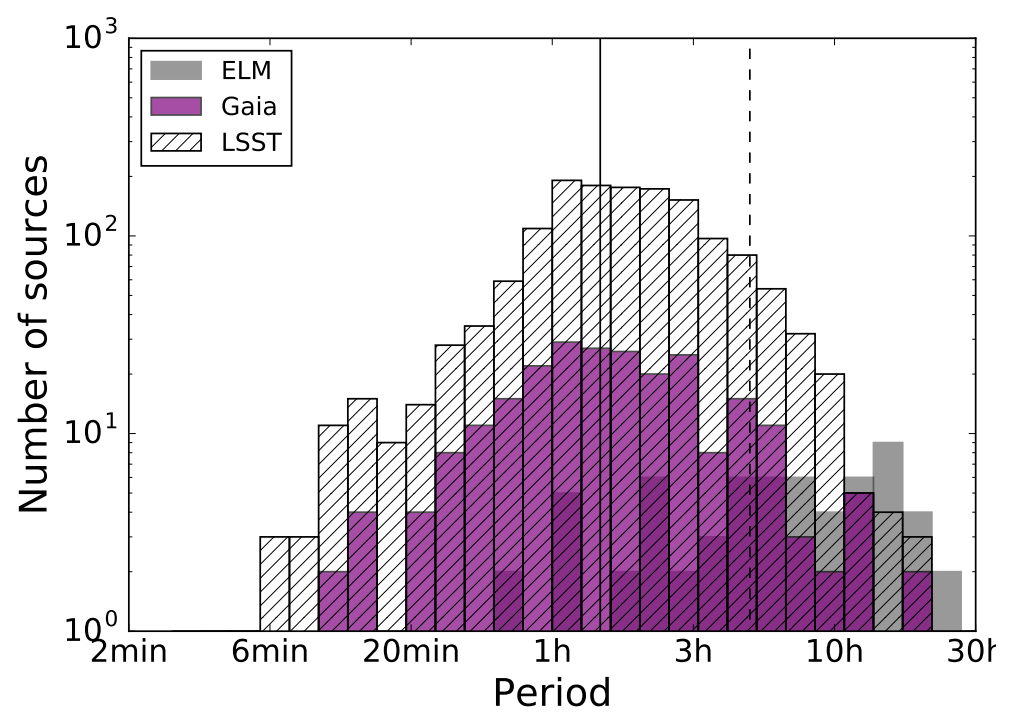

Figure 1.6 Expected LISA double WD sources for which an optical counterpart can be detected by LSST or Gaia. From Korol et al. (2017)

to maybe 25,000 individually resolved systems (Cornish and Robson, 2017; Korol et al. 2017). A small, but not negligible fraction will also be detectable with EM instruments (e.g. Littenberg et al., 2013).

The emission of GW will bring close double WD closer and eventually leads to Rochelobe overflow of the lowest-mass WD. Contrary to the case of two NS, there is a possibility for stable mass transfer (see Marsh et al. 2003). The expected properties of such interacting double WD correspond closely to the observed AM CVn systems (see Solheim 2010). These are characterised by very short orbital periods (5-60 min), helium accretion discs around WD with and very low-mass, unseen companions that need to be degenerate in order to fit in the orbits.

Some of the observed systems are expected to be individually detected by LISA and are referred to as verification binaries (e.g. Ströer and Vecchio, 2006, Shah et al., 2015, Brown et al., 2017). Between now and the launch of LISA there will be different instruments that have the potential to increase the number of verification binaries significantly, in particular Gaia and LSST (Korol et al., 2017, Breivik et al. 2017, and see Fig. 1.6. The combined data from GW and EM observations, in particular period evolution measurements, e.g. through eclipse timings, have the potential to greatly improve out understanding of the evolution into double WD and the small fraction that turns into AM CVn systems (Shah and Nelemans, 2014a b, Breivik et al., 2017)

We can expect to get information about the binary evolution leading to the formation of double WD and the initial binary populations for low-mass stars from the numbers and 
properties of the large sample of detected systems. In particular when the (chirp) masses can be measured (only in a subset of the individual systems), constraints on the common envelope and the stability of mass transfer between two WD can be inferred. Finally, the systems with measured distances (even fewer or the systems with complementary EM data) can be used to study the Galactic distribution of systems and, since some of these will be located throughout the Galaxy, the structure of the Milky Way (e.g. Korol et al. 2018).

\subsection{Conclusions}

We now really can say that GW astrophysics has started and is not just something for the future. At least for the next decade, binaries will be the key GW sources. With the plans for increased sensitivity, the prospects are excellent, not only for more detections, but also for sufficiently large samples that allow statistical studies. For binary BH we can expect many detections. At the moment, their formation is unclear and hopefully the statistical studies of the population will start to give directions on how to tackle the question of their origin. For double NS the first question is how many detections we will see in the next years. In addition, the first detection was such a rich event, with detections in all EM bands, that we will have to see how typical such an event is. In any case, the study of EM counterparts will provide valuable extra information that will make it easier to use them to study binary evolution. Indeed, if the merger rate is as high as suggested by this first detection, we may already have to go back to the drawing board for their evolution. In the long run, detectors such as LISA and ET will start the phase of mass detections, with thousands of sources expected. The WD binaries detected by LISA will provide new information on low-mass binary formation and the delicate process of mass transfer between WD. Before the LISA launch, many new systems will be detected and long-term monitoring of (eclipsing) sources will provide crucial information to complement the LISA measurements. Overall, it is clear that our understanding of binary evolution will get a boost from all these new data!

\section{References}

Aasi J., , Abbott B. P., , Abbott R., et al. 2015. Advanced LIGO. Classical and Quantum Gravity, 32(7), 074001.

Abadie J., , Abbott B. P., , Abbott R., et al. 2010. TOPICAL REVIEW: Predictions for the rates of compact binary coalescences observable by ground-based gravitational-wave detectors. Classical and Quantum Gravity, 27(17), 173001.

Abbott B. P., , Abbott R., , Abbott T. D., et al. 2016a. Astrophysical Implications of the Binary Black-hole Merger GW150914. ApJ, 818, L22.

Abbott B. P., , Abbott R., , Abbott T. D., et al. 2016b. Binary Black Hole Mergers in the First Advanced LIGO Observing Run. Physical Review X, 6(4), 041015.

Abbott B. P., , Abbott R., , Abbott T. D., et al. 2016c. GW150914: The Advanced LIGO Detectors in the Era of First Discoveries. Physical Review Letters, 116(13), 131103. 
Abbott B. P., , Abbott R., , Abbott T. D., et al. 2016d. Localization and Broadband Followup of the Gravitational-wave Transient GW150914. ApJ, 826, L13.

Abbott B. P., , Abbott R., , Abbott T. D., et al. 2016e. Observation of Gravitational Waves from a Binary Black Hole Merger. Physical Review Letters, 116(6), 061102.

Abbott B. P., , Abbott R., , Abbott T. D., et al. 2016f. Prospects for Observing and Localizing Gravitational-Wave Transients with Advanced LIGO and Advanced Virgo. Living Reviews in Relativity, 19, 1. arXiv:1304.0670.

Abbott B. P., , Abbott R., , Abbott T. D., et al. 2016g. Tests of General Relativity with GW150914. Physical Review Letters, 116(22), 221101.

Abbott B. P., , Abbott R., , Abbott T. D., et al. 2017a. A gravitational-wave standard siren measurement of the Hubble constant. Nature, 551, 85-88.

Abbott B. P., , Abbott R., , Abbott T. D., et al. 2017b. GW170104: Observation of a 50Solar-Mass Binary Black Hole Coalescence at Redshift 0.2. Physical Review Letters, 118(22), 221101.

Abbott B. P., , Abbott R., , Abbott T. D., et al. 2017c. GW170608: Observation of a 19solar-mass Binary Black Hole Coalescence. ArXiv e-prints, Nov.

Abbott B. P., , Abbott R., , Abbott T. D., et al. 2017d. GW170814: A Three-Detector Observation of Gravitational Waves from a Binary Black Hole Coalescence. Physical Review Letters, 119(14), 141101.

Abbott B. P., , Abbott R., , Abbott T. D., et al. 2017e. GW170817: Observation of Gravitational Waves from a Binary Neutron Star Inspiral. Physical Review Letters, 119(16), 161101.

Abbott B. P., , Abbott R., , Abbott T. D., et al. 2017f. Multi-messenger Observations of a Binary Neutron Star Merger. ApJ, 848, L12.

Acernese F., , Agathos M., , Agatsuma K., et al. 2015. Advanced Virgo: a secondgeneration interferometric gravitational wave detector. Classical and Quantum Gravity, 32(2), 024001.

Amaro-Seoane P., , Audley H., , Babak S., et al. 2017. Laser Interferometer Space Antenna. ArXiv e-prints, Feb.

Armstrong J. W., , Estabrook F. B., Tinto M., . 1999. Time-Delay Interferometry for Spacebased Gravitational Wave Searches. ApJ, 527, 814-826.

Aso Y., , Michimura Y., , Somiya K., et al. 2013. Interferometer design of the KAGRA gravitational wave detector. Phys. Rev. D, 88(4), 043007.

Barnes J., Kasen D., . 2013. Effect of a High Opacity on the Light Curves of Radioactively Powered Transients from Compact Object Mergers. ApJ, 775, 18.

Belczynski K., , Kalogera V., Bulik T., . 2002. A Comprehensive Study of Binary Compact Objects as Gravitational Wave Sources: Evolutionary Channels, Rates, and Physical Properties. ApJ, 572, 407-431.

Belczynski K., , Repetto S., , Holz D. E., et al. 2016a. Compact Binary Merger Rates: Comparison with LIGO/Virgo Upper Limits. ApJ, 819, 108.

Belczynski K., , Holz D. E., , Bulik T., O’Shaughnessy R., . 2016b. The first gravitationalwave source from the isolated evolution of two stars in the 40-100 solar mass range. Nature, 534, 512-515.

Bloemen S., , Groot P., , Woudt P., et al. 2016 (July). MeerLICHT and BlackGEM: custombuilt telescopes to detect faint optical transients. Page 990664 of: Ground-based and Airborne Telescopes VI. Proc. SPIE, vol. 9906.

Breivik K., , Rodriguez C. L., , Larson S. L., , Kalogera V., Rasio F. A., . 2016. Distinguishing between Formation Channels for Binary Black Holes with LISA. ApJ, 830, L18. 
Breivik K., , Kremer K., , Bueno M., et al. 2017. Characterizing accreting double white dwarf binaries with LISA and Gaia. ArXiv e-prints, Oct.

Breton R. P., , Kaspi V. M., , Kramer M., et al. 2008. Relativistic Spin Precession in the Double Pulsar. Science, 321, 104.

Brown W. R., , Kilic M., , Kosakowski A., Gianninas A., . 2017. Discovery of a Detached, Eclipsing 40 Minute Period Double White Dwarf Binary and a Friend: Implications for He+CO White Dwarf Mergers. ApJ, 847, 10.

Casares J., Jonker P. G., . 2014. Mass Measurements of Stellar and Intermediate-Mass Black Holes. Space Sci. Rev., 183, 223-252.

Chruslinska M., , Belczynski K., , Klencki J., Benacquista M., . 2017. Double neutron stars: merger rates revisited. ArXiv e-prints, Aug.

Clark J. P. A., , van den Heuvel E. P. J., Sutantyo W., . 1979. Formation of neutron star binaries and their importance for gravitational radiation. A\&A, 72, 120-128.

Cornish N., Robson T., . 2017 (May). Galactic binary science with the new LISA design. Page 012024 of: Journal of Physics Conference Series. Journal of Physics Conference Series, vol. 840 .

Cowperthwaite P. S., , Berger E., , Villar V. A., et al. 2017. The Electromagnetic Counterpart of the Binary Neutron Star Merger LIGO/Virgo GW170817. II. UV, Optical, and Near-infrared Light Curves and Comparison to Kilonova Models. ApJ, 848, L17.

De Loore C., , De Greve J. P., de Cuyper J. P., . 1975. Evolution of massive close binaries. II - The POST X-ray binary stage: Origin of run-away and binary pulsars. Ap\&SS, 36, 219-225.

de Mink S. E., Mandel I., . 2016. The chemically homogeneous evolutionary channel for binary black hole mergers: rates and properties of gravitational-wave events detectable by advanced LIGO. MNRAS, 460, 3545-3553.

Drout M. R., , Piro A. L., , Shappee B. J., et al. 2017. Light curves of the neutron star merger GW170817/SSS17a: Implications for r-process nucleosynthesis. Science, 358, $1570-1574$.

Einstein A., . 1916. Näherungsweise Integration der Feldgleichungen der Gravitation. Sitzungsberichte der Königlich Preußischen Akademie der Wissenschaften (Berlin), Seite 688-696.

Einstein A., . 1918. Über Gravitationswellen. Sitzungsberichte der Königlich Preußischen Akademie der Wissenschaften (Berlin), Seite 154-167.

eLISA Consortium, Amaro Seoane P., , Aoudia S., et al. 2013. The Gravitational Universe. ArXiv e-prints, May.

Evans C. R., , Iben Jr I., Smarr L., . 1987. Degenerate dwarf binaries as promising sources of gavitaional radiation. ApJ, 323, 129-139.

Flannery B. P., van den Heuvel E. P. J., . 1975. On the origin of the binary pulsar PSR 1913 + 16. A\&A, 39, 61-67.

Fragos T., , Willems B., , Kalogera V., et al. 2009. Understanding Compact Object Formation and Natal Kicks. II. The Case of XTE J1118 + 480. ApJ, 697, 1057-1070.

Hils D., , Bender P. L., Webbink R. F., . 1990. Gravitational radiation from the Galaxy. ApJ, 360, 75-94.

Hulse R. A., Taylor J. H., . 1975. Discovery of a pulsar in a binary system. ApJ, 195, L51-L53.

Iyer B., et al. 2011. LIGO India. Tech. rept. LIGO-M1100296. https://dcc.ligo.org/LIGOM1100296/public.

Jonker P. G., Nelemans G., . 2004. The distances to Galactic low-mass X-ray binaries: consequences for black hole luminosities and kicks. MNRAS, 354, 355. 
Kasliwal M. M., , Nakar E., , Singer L. P., et al. 2017. Illuminating gravitational waves: A concordant picture of photons from a neutron star merger. Science, 358, 1559-1565.

Korol V., , Rossi E. M., , Groot P. J., et al. 2017. Prospects for detection of detached double white dwarf binaries with Gaia, LSST and LISA. MNRAS, 470, 1894-1910.

Korol V., , Rossi E. M., Barausse E., . 2018. A multi-messenger study of the Milky Way's stellar disc and bulge with LISA, Gaia and LSST. ArXiv e-prints, June.

Kulkarni S. R., . 2005. Modeling Supernova-like Explosions Associated with Gamma-ray Bursts with Short Durations. ArXiv Astrophysics e-prints, Oct.

Levan A. J., , Lyman J. D., , Tanvir N. R., et al. 2017. The Environment of the Binary Neutron Star Merger GW170817. ApJ, 848, L28.

Li L.-X., Paczyński B., . 1998. Transient Events from Neutron Star Mergers. ApJ, 507, L59-L62.

Littenberg T. B., , Larson S. L., , Nelemans G., Cornish N. J., . 2013. Prospects for observing ultracompact binaries with space-based gravitational wave interferometers and optical telescopes. MNRAS, 429, 2361-2365.

Lyne A. G., Lorimer D. R., . 1994. HIGH BIRTH VELOCITIES OF RADIO PULSARS. Nat, 369, 127-.

Mapelli M., . 2016. Massive black hole binaries from runaway collisions: the impact of metallicity. MNRAS, 459, 3432-3446.

Marsh T. R., . 2011. Double white dwarfs and LISA. Classical and Quantum Gravity, 28(9), 094019.

Marsh T. R., , Nelemans G., Steeghs D., . 2003. Direct Impact Accretors: Evolutionary links between detached and semi-detached white dwarfs. Page 275 of: de Martino D., , Kalytis R., , Silvotti R., Solheim J., (eds), White Dwarfs, Proc. XIII Workshop on White Dwarfs. Kluwer.

Massevitch A. G., , Tutukov A. V., Iungelson L. R., . 1976. Evolution of massive close binaries and formation of neutron stars and black holes. Ap\&SS, 40, 115-133.

Metzger B. D., . 2017. Kilonovae. Living Reviews in Relativity, 20, 3.

Nelemans G., , Tauris T. M., van den Heuvel E. P. J., . 1999. Constraints on mass ejection in black hole formation derived from black hole X-ray binaries. A\&A, 352, L87-L90.

Nelemans G., , Yungelson L. R., Portegies Zwart S. F., . 2001. The gravitational wave signal from the Galactic disk population of binaries containing two compact objects. A\&A, 375, 890-898.

Paczyński B., . 1967. ? Acta Astron., 17, 287.

Park D., , Kim C., , Lee H. M., , Bae Y.-B., Belczynski K., . 2017. Black hole binaries dynamically formed in globular clusters. MNRAS, 469, 4665-4674.

Peters P. C., . 1964. Gravitational Radiation and the Motion of Two Point Masses. Physical Review, 136, 1224-1232.

Peters P. C., Matthews J., . 1963. .. Phys. Rev., 131, 435.

Phinney E. S., . 1991. The rate of neutron star binary mergers in the universe - Minimal predictions for gravity wave detectors. ApJ, 380, L17-L21.

Pian E., , D’Avanzo P., , Benetti S., et al. 2017. Spectroscopic identification of r-process nucleosynthesis in a double neutron-star merger. Nature, 551, 67-70.

Podsiadlowski P., , Langer N., , Poelarends A. J. T., et al. 2004. The Effects of Binary Evolution on the Dynamics of Core Collapse and Neutron Star Kicks. ApJ, 612, 1044-1051.

Portegies Zwart S. F., McMillan S. L. W., . 2000. Black Hole Mergers in the Universe. ApJ, 528, L17-L20.

Press W., Thorne K. S., . 1972. Gravitational-Wave Astronomy. ARA\&A, 10, 335-374. 
Repetto S., Nelemans G., . 2015. Constraining the formation of black holes in short-period black hole low-mass X-ray binaries. MNRAS, 453, 3341-3355.

Repetto S., , Davies M. B., Sigurdsson S., . 2012. Investigating stellar-mass black hole kicks. MNRAS, 425, 2799-2809.

Repetto S., , Igoshev A. P., Nelemans G., . 2017. The Galactic distribution of X-ray binaries and its implications for compact object formation and natal kicks. MNRAS, 467, 298310.

Rodriguez C. L., , Chatterjee S., Rasio F. A., . 2016. Binary black hole mergers from globular clusters: Masses, merger rates, and the impact of stellar evolution. Phys. Rev. D, 93(8), 084029.

Sathyaprakash B., , Abernathy M., , Acernese F., et al. 2012. Scientific objectives of Einstein Telescope. Classical and Quantum Gravity, 29(12), 124013.

Sathyaprakash B. S., Schutz B. F., . 2009. Physics, Astrophysics and Cosmology with Gravitational Waves. Living Reviews in Relativity, 12, 2.

Schutz B. F., . 1989. Gravitational wave sources and their detectability. Classical and Quantum Gravity, 6, 1761-1780.

Sesana A., . 2016. Prospects for Multiband Gravitational-Wave Astronomy after GW150914. Physical Review Letters, 116(23), 231102.

Shah S., Nelemans G., . 2014a. Constraining Parameters of White-dwarf Binaries Using Gravitational-wave and Electromagnetic Observations. ApJ, 790, 161.

Shah S., Nelemans G., . 2014b. Measuring Tides and Binary Parameters from Gravitational Wave Data and Eclipsing Timings of Detached White Dwarf Binaries. ApJ, 791, 76.

Shah S., , Larson S. L., Brown W., . 2015 (May). Ultra-compact binaries as gravitational wave sources. Page 012003 of: Journal of Physics Conference Series. Journal of Physics Conference Series, vol. 610.

Sigurdsson S., Hernquist L., . 1993. Primordial black holes in globular clusters. Nature, 364, 423-425.

Smartt S. J., , Chen T.-W., , Jerkstrand A., et al. 2017. A kilonova as the electromagnetic counterpart to a gravitational-wave source. Nature, 551, 75-79.

Solheim J.-E., . 2010. AM CVn Stars: Status and Challenges. PASP, 122, 1133-1163.

Ströer A., Vecchio A., . 2006. The LISA verification binaries. Classical and Quantum Gravity, 23, 809.

Sukhbold T., , Ertl T., , Woosley S. E., , Brown J. M., Janka H.-T., . 2016. Core-collapse Supernovae from 9 to 120 Solar Masses Based on Neutrino-powered Explosions. ApJ, 821, 38.

Tauris T. M., , Kramer M., , Freire P. C. C., et al. 2017. Formation of Double Neutron Star Systems. ApJ, 846, 170.

Taylor J. H., Weisberg J. M., . 1982. A new test of general relativity - Gravitational radiation and the binary pulsar PSR 1913+16. ApJ, 253, 908-920.

Thorne K. S., . 1997. Gravitational radiation: a new window onto the universe. $R v M A, \mathbf{1 0}$, $1-28$.

Toonen S., , Nelemans G., Portegies Zwart S., . 2012. Supernova Type Ia progenitors from merging double white dwarfs. Using a new population synthesis model. A\&A, 546, A70.

Tutukov A. V., Yungelson L. R., . 1979. "On the influence of emission of gravitational waves on the evolution of low-mass close binary stars". Acta Astron., 29, 665-680.

Ugliano M., , Janka H.-T., , Marek A., Arcones A., . 2012. Progenitor-explosion Connection and Remnant Birth Masses for Neutrino-driven Supernovae of Iron-core Progenitors. ApJ, 757, 69. 
van den Heuvel E. P. J., De Loore C., . 1973. The nature of X-ray binaries III. Evolution of massive close binaries with one collapsed component - with a possible application to Cygnus X-3. A\&A, 25, 387-395.

van den Heuvel E. P. J., , Portegies Zwart S. F., de Mink S. E., . 2017. Forming shortperiod Wolf-Rayet X-ray binaries and double black holes through stable mass transfer. MNRAS, 471, 4256-4264.

Verbunt F., , Igoshev A., Cator E., . 2017. The observed velocity distribution of young pulsars. ArXiv e-prints, Aug.

Weiss R., . 1972. Electromagnetically coupled broadband gravitational wave antenna. Quarterly Progress Report, Research Laboratory of Electronics of MIT, 105, 54.

White N. E., van Paradijs J., . 1996. The galactic distribution of black hole candidates in low-mass X-ray binary systems. ApJ, 473, L25-L29.

Willems B., , Henninger M., , Levin T., et al. 2005. Understanding Compact Object Formation and Natal Kicks I. Calculation Methods and the case of GRO J1655-40. ApJ, 625, 324 . 